\title{
Critical appraisal of rituximab in the maintenance treatment of advanced follicular lymphoma
}

This article was published in the following Dove Press journal:

Cancer Management and Research

27 October 2015

Number of times this article has been viewed

\section{David Aguiar-Bujanda \\ María Jesús Blanco-Sánchez \\ María Hernández-Sosa \\ Saray Galván-Ruíz \\ Samuel Hernández- \\ Sarmiento \\ Department of Medical Oncology, Hospital Universitario de Gran Canaria Doctor Negrín, Las Palmas de Gran Canaria, Spain}

Correspondence: David Aguiar-Bujanda Department of Medical Oncology, Hospital Universitario de Gran Canaria Doctor Negrín, C/Barranco de la ballena s/n, 350I0 Las Palmas de Gran Canaria, Spain

Tel +34928450400

Fax +34928450079

Email dagubuj@gobiernodecanarias.org

\begin{abstract}
Rituximab is an $\operatorname{IgG1}$, chimeric monoclonal antibody specifically designed to recognize the CD20 antigen expressed on the surface of normal and malignant B-lymphocytes, from the B-cell precursor to the mature B-cells of the germinal center, and by most neoplasms derived from B-cells. After 2 decades of use, rituximab is firmly positioned in the treatment of follicular lymphoma (FL), both in the front line and in the relapsing disease, improving previous results by including it in classical chemotherapy regimens. However, the pharmacology of rituximab continues to generate controversial issues especially regarding the mechanisms of action in vivo. The contribution of rituximab as a maintenance treatment in FL has been significant progress in the management of this disease without an increase in side effects or a decrease in the quality of life of patients. With the widespread use of rituximab, there are new security alerts and side effects not previously detected in the pivotal trials that clinicians should learn to recognize and manage. In this article, we will review the pharmacokinetics and pharmacodynamics of rituximab, the management issues in the treatment of advanced FL focusing on maintenance rituximab, its long-term efficacy and safety profile, and its effect on the quality of life.
\end{abstract}

Keywords: follicular lymphoma, long-term efficacy, maintenance, rituximab, toxicity

\section{Introduction}

Rituximab (IDEC-C2B8; MabThera ${ }^{\circledR}$, Roche, Basel, Switzerland; Rituxan ${ }^{\circledR}$, Biogen Idec, Inc., Cambridge, MA, USA and Genentech, Inc., South San Francisco, CA, USA) is an $\operatorname{IgG} 1$, chimeric monoclonal antibody (mAb) containing murine lightand heavy-chain variable-region sequences and human constant-region sequences. Rituximab specifically recognizes the CD20 antigen expressed on the surface of normal and malignant B-lymphocytes, from the B-cell precursor to the mature B-cells of the germinal center, and by most neoplasms derived from B-cells. ${ }^{1,2}$

Rituximab was the first mAb approved by the US Food and Drug Administration in 1997 and since then has become widely used for a variety of neoplastic and autoimmune conditions. Rituximab is part of the standard treatment of patients with B-cell non-Hodgkin's lymphoma (NHL), including follicular lymphoma (FL), diffuse large B-cell lymphoma, and small lymphocytic lymphoma/chronic lymphocytic leukemia, and for the treatment of rheumatoid arthritis, granulomatosis with polyangiitis and microscopic polyangiitis. Other off-label uses include Hodgkin's lymphoma, mantle cell lymphoma, marginal zone lymphoma, idiopathic thrombocytopenic purpura, multiple sclerosis, pemphigus vulgaris unresponsive to standard therapy, steroid-refractory chronic graft-versus-host disease, and many other autoimmune disorders. ${ }^{3,4}$ 
FL is the second most frequent type of lymphoma, with an increasing incidence especially in Western countries. ${ }^{5,6}$ Approximately $80 \%$ of patients with FL present with advanced stage at diagnosis. Clinically, FL is usually characterized by a nonaggressive course, with a slow increase of painless lymph nodes, sometimes with fluctuations in size for several years, and many patients remain asymptomatic despite progressive disease. FL is divided in three distinct grades according to the WHO classification, namely grade 1 , grade 2 , and grade 3 . The grade 3 is further divided into grade $3 \mathrm{~A}$ and grade $3 \mathrm{~B}$, the latter usually exhibiting an aggressive course similar to that of diffuse large B-cell lymphoma, for what the general recommendation is to follow a therapeutic approach similar to that used for this type of lymphoma. ${ }^{6}$

The fact that, most patients with advanced FL show a continuous pattern of relapse during years despite an excellent response to therapy, and that the duration of response gets shorter after every relapse, have made that FL has been considered an incurable illness. The prognosis of FL remained stable for decades, with an overall survival (OS) of 10 years; however, an increase in OS has been observed in the last 2 decades, which currently reaches and exceeds 15 years. ${ }^{7}$ This progress has been achieved in part through the introduction of rituximab as a cornerstone of therapy.

In this article, we review the pharmacokinetics (PK) and pharmacodynamics of rituximab, the management issues in the treatment of advanced FL focusing on maintenance rituximab (MR), its long-term efficacy and safety profile, and its effect on the quality of life (QoL).

\section{Rituximab: mechanisms of action}

Rituximab responds specifically to the CD20 antigens found on the surface of malignant and normal B-cells, and is able to recognize it with an affinity of approximately $5.2 \times 10^{-9} \mathrm{M}^{2}$ The accurate in vivo role of CD20 is still to a great extent unknown. It is suggested that the CD20 antigen may regulate the process of B-cell differentiation. ${ }^{8}$ Some data indicate that CD20 is a potential ion channel, playing an important role in $\mathrm{Ca}^{2+}$ influx across plasma membranes, and may be involved in the regulation of signal transduction allowing activation of B-cells. ${ }^{9}$ The precise in vivo mechanisms of action of rituximab are not fully clarified. A number of antitumor effects have been suggested, including antibody-dependent cellular cytotoxicity (ADCC), complement-dependent cytotoxicity (CDC), induction of direct cell death, and sensitization of B-cells to chemotherapy (CT).

ADCC is mediated through ligation and activation of the Fc portion of rituximab to the Fc receptors that are expressed on natural killer (NK) cells, macrophages, monocytes, and dendritic cells. ${ }^{10} \mathrm{NK}$ cells recognize rituximab opsonized tumor cells and mediate cell lysis through the release of cytotoxic substances. On the other hand, monocytes and macrophages promote phagocytic killing of the rituximabcoated malignant cells. ${ }^{11}$ The binding affinity of Fc receptors to rituximab may be influenced by genomic polymorphisms in Fc $\gamma$ RIIIa genes, influencing in the response rates to rituximab and survival. Polymorphisms in Fc $\gamma$ RIIIa expressing either valine (V) or phenylalanine (F) at the 158 position conditions the attachment of the antibody, being stronger to the homozygous Fc $\gamma$ RIIIa-158V (V/V) than to the homozygous F/F or to the heterozygous $\mathrm{F} / \mathrm{V}$ forms. ${ }^{12,13}$ As a result, more significant response to rituximab and higher median survival have been observed in patients with the $158 \mathrm{~V}$ allotype. ${ }^{14,15}$ Finally, stimulation and expansion of $\mathrm{NK}$ and macrophages with cytokines like interleukin 2 (IL-2), IL-12, or granulocyte-macrophage colony-stimulating factor may enhance the ADCC of rituximab as shown in different studies. ${ }^{16}$

Rituximab promotes CDC by the activation of the classical complement pathway. The Fc component binds to the $\mathrm{C} 1 \mathrm{q}$, triggering a proteolytic cascade that results in the liberation of $\mathrm{C} 3 \mathrm{~b}$ and ultimately into the generation of membrane attack complex. ${ }^{10}$ It is postulated that the complement cascade may be involved in the first infusion symptoms suffered from some patients, and in the rapid B-cell depletion observed following the initial dose of antibody. ${ }^{17,18}$ Nevertheless the exact in vivo contribution of CDC to the cytotoxic effect of rituximab is still controversial.

Another potential mechanism of action involves the induction of direct cell death as a consequence of CD20 ligation. The binding of rituximab triggers the inhibition of several intracellular signaling pathways associated with cell survival, such as $\mathrm{PI} 3 \mathrm{~K} / \mathrm{AKT}$, nuclear factor- $\mathrm{KB}$, the p38 mitogen-activated protein kinase, and ERK1/2. ${ }^{19}$ In addition, rituximab induces apoptosis by a caspase-independent mechanism, and by the inhibition of the antiapoptotic Bcl-2/Bcl-xL proteins. ${ }^{20}$

Recent clinical and in vitro data indicates that rituximab sensitizes malignant B cells to CT, generating a synergic effect with the cytotoxic agents by modifying the expression pattern of proteins involved in apoptosis. ${ }^{21}$ It has been suggested that when combining with rituximab, the dose of synergistic cytotoxic drugs could be lower and less toxic enough to effectively exploit this mechanism of apoptosis..$^{20,21}$

\section{Pharmacokinetics of rituximab}

Considerably, variations in the rituximab serum levels are seen among patients treated with comparable doses 
by intravenous (IV) injections. The best model to explain rituximab $\mathrm{PK}$ is a two-compartment model, with first-order distribution kinetics between the peripheral circulation and the accessible pool of CD20-binding domains, with mean half-lives of approximately 1.3 and 19 days for distribution and elimination, respectively. ${ }^{22}$

Variability in rituximab PK may be explained in part by sex and interethnic differences, number of circulating CD20+ cells, intensity of CD20 expression on tumoral cells, and tumor burden. However, Mangel et al suggest that the PK profile of rituximab is not necessarily correlated with tumor burden. They found that PK parameters were not different between patients with significant tumor burden and those in clinical response or minimal disease, and that after four injections the final serum rituximab concentrations were similar in both situations. ${ }^{23}$

Rituximab PK is linear, with proportional increases in peak concentrations $\left(\mathrm{C}_{\max }\right)$ with each infusion. $\mathrm{PK}$ of rituximab were first described in a Phase I clinical trial of relapsed low-grade B-cell lymphoma patients treated with a single IV infusion of $10,50,100,250$, or $500 \mathrm{mg} / \mathrm{m}^{2}$. The serum half-life of the free antibody at the doses of 100, 250, and $500 \mathrm{mg} / \mathrm{m}^{2}$ was 4.4 days, ranging from 1.6 to 10.5 days. In six of nine patients, the levels of rituximab detected after 14 days were $>10 \mu \mathrm{g} / \mathrm{mL}$. A fast and specific reduction of CD20+ B cells was observed between 24 and 72 hours in the peripheral blood of patients who received doses of $100 \mathrm{mg} / \mathrm{m}^{2}$ or greater, lasting a minimum of $2-3$ months in most of them. ${ }^{24}$ A subsequent Phase I study with multiple weekly infusions of rituximab at 125,250 , and $375 \mathrm{mg} / \mathrm{m}^{2}$, showed that the $\mathrm{C}_{\max }$ for both the first and fourth infusions increased with increasing dose, and that the $\mathrm{C}_{\max }$ and serum half-life increased between the first and fourth infusions for most patients. ${ }^{25}$ The dose of $375 \mathrm{mg} / \mathrm{m}^{2}$ was selected for further clinical trials in patients with FL or indolent, relapsed B-cell lymphoma.

PK analysis performed in the pivotal Phase III trial at the dose of $375 \mathrm{mg} / \mathrm{m}^{2}$, showed that the serum concentrations increased with each infusion, with a median $\mathrm{C}_{\max }$ being doubled from 205.6 to $464.7 \mu \mathrm{g} / \mathrm{mL}$ from the first to the fourth infusion, with a corresponding increase in the halfelimination from 76.3 to 205.8 hours. An inverse correlation was found between the mean serum levels of rituximab with both, the tumor bulk measure and the baseline number of circulating B cells. Most of the patients had measurable levels of rituximab at 3 months of last infusion, and some of them even at 6 months. Higher serum rituximab concentrations were associated with better clinical response. At 3 months posttreatment, median serum levels in responsive patients were 25.4 versus $5.9 \mu \mathrm{g} / \mathrm{mL}$ in nonresponders. ${ }^{26,27}$

An extended rituximab schedule consisting of eight weekly infusions led to similar results in a Phase II trial. The response was strongly correlated with serum concentrations of rituximab both during and posttreatment. The responder patients showed higher serum concentrations of rituximab compared with those who did not respond. Although the median preinfusion serum concentrations of rituximab increased with each infusion, a plateau (range $518.1-558.1 \mu \mathrm{g} / \mathrm{mL}$ ) on median postinfusion serum levels was observed after the sixth infusion. ${ }^{28}$

As mentioned above, the pivotal trial of rituximab established $25 \mu \mathrm{g} / \mathrm{mL}$ as the minimum therapeutic threshold to maintain over time. ${ }^{27}$ Different schedules of MR have been employed to obtain this minimum rituximab level after the induction phase and improve therapeutic results. Rituximab has been administered in different schedules, the most used being: one weekly infusion over 4 weeks repeated every 6 months, and a single infusion every 2 or 3 months for 2 years. At this time, the best MR schedule has not been established in a randomized trial, but the data from several studies make reasonable to assume that the administration of MR every 2 months achieves the optimal rituximab serum levels.

A more convenient subcutaneous (SC) formulation of rituximab has been developed and is now being tested under clinical trials. Rituximab is typically administered by IV infusions over 1.5-6 hours, and thus a SC rituximab administration over 5-6 minutes could increase patient convenience, improve cost-effectiveness, and reduce adverse events. ${ }^{29}$ As a result of a lower absorption, the dose of rituximab must be increased when administered by SC injection, and thus larger volumes of drug are needed. Such SC injection is possible by increasing 12-fold concentration of rituximab respecting the IV preparation, and by the addition of the enzyme recombinant human hyaluronidase (rHuPH20). ${ }^{30} \mathrm{SC}$ rituximab is concentrated at $120 \mathrm{mg} / \mathrm{mL}$ compared with the IV formulation of $10 \mathrm{mg} / \mathrm{mL}$, and $\mathrm{rHuPH} 20$ transiently degrades interstitial hyaluronan at the injection site, increasing the volume that can be administered and facilitating drug entry into the circulation. ${ }^{31}$ In the two-stage, Phase IB SparkThera study comparing the standard IV dose of $375 \mathrm{mg} / \mathrm{m}^{2}$ with a fixed SC dose of $1,400 \mathrm{mg}$ in the maintenance treatment of FL, it was confirmed the noninferior serum through concentration levels of the SC formulation, with no differences in the toxicity profile. ${ }^{32}$ The results from the stage I analysis of the randomized Phase III SABRINA study show that the PK profile of SC rituximab at a fixed dose of 1,400 mg 
was noninferior to IV rituximab in terms of serum through concentrations, and no new safety signals were described. After stage I, the patients will continue to receive SC or IV MR for up to 2 years. ${ }^{33}$ The stage II of this trial will provide safety and efficacy data of the SC administration.

\section{Management issues in the treatment of advanced FL}

The clinical evaluation of a patient with FL must take into consideration two different aspects to decide the optimal treatment. The patients are classified as limited stage when the disease fits with the definition of stage I or II according to the Ann Arbor staging system. The patients with stage III-IV and those with symptomatic or voluminous disease are better classified as advanced stage, which is further divided into high-tumor burden (HTB) or low-tumor burden (LTB), depending on the presence of symptoms and factors related to the tumor load. There are several sets of criteria to assign a patient with advanced FL to either the HTB or the LTB group, but currently the most accepted are the GELF criteria (from the Groupe pour l'Etude de Lymphome Folliculaire). ${ }^{34}$

\section{Asymptomatic patients with an LTB}

Because of the indolent course of FL and the lack of a curative treatment, the initial therapeutic approach of patients with advanced LTB-FL has been the watchful waiting (WW) strategy. Older studies demonstrated that when compared to WW, a prompt start of therapy prolongs the time to next treatment and the progression-free survival (PFS), but no change in OS. ${ }^{35,36}$ The WW strategy can avoid the administration of a toxic treatment in approximately $20 \%$ of patients with LTB-FL, while this percentage may be greater in aging patients. ${ }^{35-38}$ Two modern randomized studies, the Intergroup study ${ }^{39}$ and the RESORT trial, ${ }^{40}$ have randomized patients with LTB to WW or rituximab. In this patient population, treatment with rituximab prolongs PFS and time to next treatment, and produces higher responses compared with the WW strategy. Nevertheless, the OS is not jeopardized if WW is indicated despite the use of rituximab. For these reasons, there is still debated whether to initiate immediate treatment or delay it in a patient with asymptomatic LTB-FL. An alternative approach in case of patients with troubles coping to the WW option, and for patients that would not tolerate more aggressive treatments in the event of need, can be the administration of weekly rituximab for 4 weeks as in the RESORT trial, followed by observation and retreatment at progression. ${ }^{40}$

\section{Symptomatic patients with HTB}

Patients with advanced HTB-FL are generally treated with CT or rituximab plus CT (R-CT) combinations. The incorporation of rituximab to $\mathrm{CT}$ regimens for FL has resulted in better outcomes, improving response rates and survival for both untreated (Table 1) and relapsed patients. In the first-line setting, R-CT has shown to be superior over CT in at least four randomized trials (Table 1), with better results for the rituximab combinations in terms of response rate/complete response (CR), PFS, event-free survival (EFS), time to treatment failure, and most importantly OS. ${ }^{41-44}$

Several R-CT regimens have been tested, the most frequently applied are: R-CHOP (rituximab, cyclophosphamide,

Table I Results from randomized Phase III trials of induction chemotherapy plus rituximab in the first-line treatment of follicular lymphoma

\begin{tabular}{|c|c|c|c|c|c|c|}
\hline Study & $\mathbf{n}$ & Regimen & RR (\%) & CR (\%) & TTF/PFS/EFS & OS \\
\hline \multirow[t]{2}{*}{ Hiddemann et al ${ }^{4 l}$} & 428 & CHOP & 90 & 17 & $3-y$ TTF $50 \%$ & $3-y 86 \%$ \\
\hline & & $\mathrm{R}-\mathrm{CHOP}$ & $96^{a}$ & 20 & 3-y TTF 75\%a & $3-y 95 \%$ \\
\hline \multirow[t]{2}{*}{ Herold et al ${ }^{42}$} & 201 & $M C P+I$ & 75 & 25 & 4-y PFS 40\% & $4-y 74 \%$ \\
\hline & & $R-M C P+I$ & $92^{\mathrm{a}}$ & $50^{\mathrm{a}}$ & $4-y$ PFS 7I\%a & $4-y 87 \%$ a \\
\hline \multirow[t]{2}{*}{ Marcus et al ${ }^{43}$} & 321 & CVP & 57 & 10 & TTF 7 m & $4-y 77 \%$ \\
\hline & & R-CVP & $81^{a}$ & $4 l^{a}$ & TTF $27 \mathrm{~m}^{\mathrm{a}}$ & $4-y 83 \%$ a \\
\hline \multirow[t]{2}{*}{ Salles et $\mathrm{a}{ }^{44}$} & 358 & $\mathrm{CHVP}+\mathrm{I}$ & 85 & 34 & $5-y$ EFS $37 \%$ & $5-y 79 \%$ \\
\hline & & $\mathrm{R}-\mathrm{CHVP}+\mathrm{I}$ & $94^{a}$ & $63^{\mathrm{a}}$ & $5-y$ EFS $53 \%^{a}$ & $5-y 84 \%^{a}$ \\
\hline \multirow[t]{3}{*}{ Federico et $\mathrm{al}^{45}$} & 534 & R-CVP & 88 & 67 & 3-y TTF $46 \%$ & $3-y 95 \%$ for \\
\hline & & $\mathrm{R}-\mathrm{CHOP}$ & 93 & 73 & $3-y \operatorname{TTF} 62 \%^{a}$ & the whole series \\
\hline & & R-FM & 91 & 72 & 3-y TTF 59\%a & \\
\hline \multirow[t]{2}{*}{ Rummel et $\mathrm{al}^{46}$} & 549 & $\mathrm{R}-\mathrm{CHOP}$ & 91 & 30 & PFS $31.2 \mathrm{~m}$ & $4-y 82 \%$ \\
\hline & & B-R & 93 & $40^{\mathrm{a}}$ & PFS $69.5 \mathrm{~m}^{\mathrm{a}}$ & $4-y 84 \%$ \\
\hline
\end{tabular}

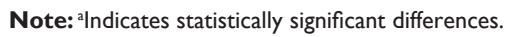

Abbreviations: B-R, bendamustine and rituximab; CHOP, cyclophosphamide, doxorubicin, vincristine, and prednisone; CHVP, cyclophosphamide, doxorubicin, etoposide, and prednisone; CR, complete response; CVP, cyclophosphamide, vincristine, and prednisone; EFS, event-free survival; I, interferon; m, months; FM, fludarabine and mitoxantrone; MCP, mitoxantrone, chlorambucil, and prednisone; $n$, number of patients; OS, overall survival; PFS, progression-free survival; R, rituximab; RR, response rate; TTF, time to treatment failure; $y$, years. 
doxorubicin, vincristine, and prednisone), R-CVP (rituximab, cyclophosphamide, vincristine, and prednisone), and B-R (bendamustine and rituximab). Because of their significant risk of toxicity (mainly hematological and infections) and higher rates of second cancers, fludarabine-based combinations are falling into disuse. ${ }^{45}$ On the other hand, the B-R regimen is increasingly being more used since it was shown to be superior over R-CHOP in the NHL 1-2003 study (Table 1) increasing the CR rates and PFS (hazard ratio [HR]: 0.58; 95\% CI: $0.44-0.74 ; P<0.0001)$, with better tolerance. ${ }^{46}$

\section{Postinduction treatments: MR}

Despite good results with front-line CT in patients with advanced FL, relapses are not infrequent, and two strategies were developed in an attempt to reduce relapses and improve survival; namely consolidation and maintenance.

Radioimmunotherapy ${ }^{47,48}$ and stem cell transplantation ${ }^{49,50}$ after first-line treatment have been used as consolidation of response with different results. However, at this time there is not an established indication for consolidation after an adequate R-CT combination given in the first-line, for what their use should be limited to clinical trials until a clearer proven benefit. $^{51}$

Interferon- $\alpha$ and rituximab were subsequently developed as maintenance treatments after induction CT. ${ }^{52,53}$ Rituximab was promptly adopted as a maintenance therapy due to a better toxicity profile, higher response rates, and longer half-life compared with interferon- $\alpha$. The benefit of MR has been demonstrated in several clinical trials for both untreated and relapsed patients (Table 2). The standard dose $\left(375 \mathrm{mg} / \mathrm{m}^{2}\right)$ of rituximab for maintenance has been used in different schedules as outlined in Table 2, although one dose every 2 months for 2 years is the preferred one in the first-line setting.

After an induction regimen (with CT or R-CT), MR has produced significant improvements in the results of untreated patients with advanced FL compared with observation in four Phase III randomized trials, ${ }^{15,54-58}$ in terms of response rate, EFS, or PFS, but without significant differences in OS or toxicity (see Table 2 for more details about characteristics of the studies and patient population).

In the SAKK $35 / 98$ study ${ }^{15,54}$ with a median follow-up of 9.5 years, the median EFS was 24 months in the MR arm versus 13 months in the observational arm $(P<0.001)$ with no relevant increase in toxicity. There was a nonsignificant difference in OS favoring the MR arm (68\% versus 54\%; HR: 0.63; 95\% CI: $0.37-1.06 ; P=0.081)$. Previously untreated patients responding to induction therapy obtained the most benefit from MR, with 8-year EFS of 45\%. ${ }^{15}$

In the ECOG 1496 study, ${ }^{55}$ patients with FL after MR showed an increase in the CR compared with those who were randomized to observation ( $37 \%$ versus $16 \%)$, with a better 3 -year PFS (64\% versus 33\%; HR: 0.4; $P<0.001$ ) and higher median PFS (4.3 versus 1.3 years; HR: 0.4; 95\% CI: 0.3-0.5; $\left.P=4.4 \times 10^{-10}\right)$. Although 3-year OS was not different in both arms, there was a trend favoring the MR arm in FL patients with $\operatorname{HTB}(P=0.03)$.

The PRIMA study ${ }^{56}$ aimed to evaluate for the first time the role of MR after induction R-CT (three different regimens allowed) in untreated patients with advanced FL.

Table 2 Randomized Phase III trials comparing maintenance rituximab versus observation after induction therapy in follicular lymphoma

\begin{tabular}{|c|c|c|c|c|c|c|c|}
\hline Study & Disease setting & $\mathbf{n}$ & Induction regimen & MR schedule & MR duration & $\begin{array}{l}\text { EFS/PFS } \\
\text { MR versus OB }\end{array}$ & $\begin{array}{l}\text { OS } \\
\text { MR versus OB }\end{array}$ \\
\hline Ghielmini et $\mathrm{al}^{54}$ & Untreated/relapsed FL & 202 & $\mathrm{R} w \mathrm{wk} \times 4$ & Once every $2 \mathrm{~m}$ & $8 \mathrm{~m}$ & 9.5-y EFS & $9.5-y$ \\
\hline Martinelli et al $^{15}$ & & & & & & 24 versus $13 \mathrm{~m}^{\mathrm{a}}$ & $68 \%$ versus $54 \%$ \\
\hline Hochster et $\mathrm{al}^{55}$ & Untreated FL & 282 & CVP $\times 6-8$ & $\begin{array}{l}\text { Once } w \mathrm{k} \times 4 \\
\text { every } 6 \mathrm{~m}\end{array}$ & $2 y$ & $\begin{array}{l}3-y \text { PFS } \\
64 \% \text { versus } 33 \%^{a}\end{array}$ & $\begin{array}{l}3-y \\
91 \% \text { versus } 86 \%\end{array}$ \\
\hline Salles et $\mathrm{al}^{56,57}$ & Untreated FL & 1,217 & $\begin{array}{l}\text { R-CVP } \times 8 \\
\text { R-CHOP } \times 6 \\
\text { R-FCM } \times 6\end{array}$ & Once every $2 \mathrm{~m}$ & $2 y$ & $\begin{array}{l}6-y \text { PFS } \\
59 \% \text { versus } 43 \% \text { a }\end{array}$ & $\begin{array}{l}6-y \\
89 \% \text { versus } 87 \%\end{array}$ \\
\hline Vitolo et $\mathrm{al}^{58}$ & Untreated FL & 234 & $\begin{array}{l}\text { R-FND } \times 4 \text { followed } \\
\text { by } R \text { wk } \times 4\end{array}$ & Once every $2 \mathrm{~m}$ & $8 \mathrm{~m}$ & $\begin{array}{l}2-y \text { PFS } \\
81 \% \text { versus } 69 \%\end{array}$ & $\begin{array}{l}3-y \text { whole series } \\
89 \%\end{array}$ \\
\hline Forstpointner et $\mathrm{al}^{59,62}$ & Relapsed FL/MCL & $319^{b}$ & $\begin{array}{l}\text { R-FCM } \times 4 \\
F C M \times 4\end{array}$ & $\begin{array}{l}\text { Once } w \mathrm{k} \times 4 \\
\text { at } 3 \text { and } 9 \mathrm{~m}\end{array}$ & $9 \mathrm{~m}$ & $\begin{array}{l}2-y P^{\circ} S^{c} \\
n r \text { versus } 26 \mathrm{~m}^{\mathrm{a}}\end{array}$ & $\begin{array}{l}3-y \\
77 \% \text { versus } 57 \%\end{array}$ \\
\hline van Oers et $a^{60,61}$ & Relapsed FL & 466 & $\begin{array}{l}\mathrm{R}-\mathrm{CHOP} \times 6 \\
\mathrm{CHOP} \times 6\end{array}$ & Once every $3 \mathrm{~m}$ & $2 y$ & $3.7 \mathrm{y}$ versus $1.3 \mathrm{y}^{\mathrm{a}}$ & $\begin{array}{l}5-y \\
74 \% \text { versus } 64 \%\end{array}$ \\
\hline
\end{tabular}

Notes: andicates statistically significant differences; ${ }^{\text {II }} 3 \mathrm{FL}$ randomized to MR versus OB; ' data for FL patients only.

Abbreviations: CHOP, cyclophosphamide, doxorubicin, vincristine, and prednisone; CVP, cyclophosphamide, vincristine, and prednisone; EFS, event-free survival; FCM, fludarabine, cyclophosphamide, and mitoxantrone; FL, follicular lymphoma; FND, fludarabine, mitoxantrone, and dexamethasone; $\mathrm{m}$, months; MCL, mantle cell lymphoma; $\mathrm{MR}$, maintenance rituximab; n, number of patients; nr, not reached; OB, observation; OS, overall survival; PFS, progression-free survival; R, rituximab; wk, weekly; $y$, years. 
Responding patients to induction R-CT were randomized to MR or observation. The 3-year PFS was better for patients randomized to $\mathrm{MR}(74.9 \%$ versus $57.6 \%$; HR: $0.55 ; 95 \%$ CI: $0.44-0.68 ; P<0.0001)$. The proportion of patients in $\mathrm{CR}$ at 2 years after randomization was also higher in the MR arm $(71.5 \%$ versus $52.2 \% ; P=0.0001)$, without a significant advantage in OS. This advantage in PFS was maintained after 6-year follow-up (59.2\% versus 42.7\%; HR: 0.58 ; 95\% CI: $0.48-0.69 ; P<0.0001) .{ }^{57}$ Despite the prolonged duration of treatment in the MR group, there were no new safety alerts, and no negative effect on subsequent therapies was observed.

Another study published by the Fondazione Italiana Linfomi raised some concerns about the benefits of MR in FL. ${ }^{58}$ After randomization, the 2-year PFS was not significantly different between the MR and the observation arms ( $81 \%$ versus $69 \% ; P=0.226$ ). This study has been criticized for the small number of patients included (limited to older than 60 years), and for the short duration of the induction and MR treatments, although it can serve to point out that MR should be used after an induction R-CT combination tested in a Phase III trial.

In conclusion, after an R-CT induction treatment, the most robust results for MR and the obtained only in a randomized Phase III trial are after R-CHOP (PRIMA trial). In the meantime, MR studies after the B-R combination are eagerly awaited.

\section{Relapsed or refractory FL: rituximab induction and maintenance}

In the relapsed/refractory (R/R) setting, induction R-CT and MR have also improved results over standard CT. In a Phase III study from the Germ Low Grade Lymphoma Study Group (GLSG), the R-FCM combination (rituximab, fludarabine, cyclophosphamide, and mitoxantrone) was superior to FCM in patients with R/R FL or MC. R-FCM was better in all subgroups, showing significant better overall response rates $(79 \%$ versus 58\%), median PFS (16 versus 10 months) and OS (2-year OS 73\% versus 53\%) than FCM alone. ${ }^{59}$ In another Phase III from the European Organization for Research and Treatment of Cancer (EORTC) 20981 trial, 465 patients with $\mathrm{R} / \mathrm{R}$ FL were randomized to R-CHOP or CHOP as an induction phase, and responders $(\mathrm{n}=334)$ were further randomized to MR every 3 months or observation. The results from the first randomization showed that the response rate, the PFS, and the OS were significantly better with R-CHOP than with CHOP ${ }^{60}$ Long-term outcome from the EORTC 20981 trial confirms that MR significantly improves median PFS versus observation (3.7 versus 1.3 years) either after R-CHOP or CHOP induction. ${ }^{61}$ No significant differences were observed between MR and observation regarding 5-year OS (74.3\% versus $64.7 \% ; P=0.07$ ), probably influenced by the use of rituximab in the salvage setting. Another two Phase III trials of MR in R/R FL have been published with similar results. Long-term results from the SAKK 35/98 study ${ }^{15}$ (untreated and $\mathrm{R} / \mathrm{R}$ patients) and the second randomization of the GLSG study ${ }^{62}$ described above (R-FCM versus FCM with or without $\mathrm{MR})$, confirm that MR after an induction regimen improves the results over observation in patients with $\mathrm{R} / \mathrm{R} \mathrm{FL}$ and should be added to standard therapy in these patients.

\section{Long-term efficacy and safety profile of rituximab}

The strongest evidence of the long-term efficacy of MR comes from the updates with longer follow-up of the SAKK 35/98 study, ${ }^{15}$ the PRIMA trial, ${ }^{57}$ and the EORTC 20981 study, ${ }^{61}$ all of them showing improvements of the results in terms of response rate, EFS, or PFS, but without benefit in OS. In the SAKK 35/98 trial, after a median follow-up of 9.5 years, EFS was 13 months in the observation group versus 24 months in the MR arm $(P<0.001)$, with nonsignificant differences in OS (54\% versus 68\%; HR: 0.63 ; 95\% CI: $0.37-1.06 ; P=0.081)$. The benefit in median EFS was greater in patients with objective response to induction therapy (3.1 versus 1.4 years) with no benefit in patients with stable disease ( 0.9 years for MR versus 0.5 years in the observation arm). Best results were in previously untreated patients with response to induction treatment (45\% patients without progression at 8 years). ${ }^{15}$ In the PRIMA trial at 6 years from randomization, PFS was $42.7 \%$ in the observational arm versus $59.2 \%$ in the MR arm (HR: 0.58 ; 95\% CI: 0.48-0.69; $P<0.0001)$. This improvement in PFS was independent of disease severity at the beginning of R-CT and of the response to induction treatment. No difference in OS was observed, with similar number of patients alive in both arms at 6 years (88.7\% in observational arm versus $87.4 \%$ in $\mathrm{MR}$ arm) ${ }^{57} \mathrm{In}$ the EORTC 20981 trial, with the follow-up of 6 years, PFS was better in the MR arm (3.7 versus 1.3 years; HR: 0.55, $P<0.001)$. As in other trials, the improvement observed in OS was not significant. ${ }^{61}$

Although rituximab is well tolerated and toxicities reported in the three studies with longer follow-up described above are consistent with the known safety profile of rituximab, there is concern about long-term effects of MR. The risk of infection has been described among patients receiving rituximab although the added risk of infection with the 
incorporation of rituximab to CT regimens for FL seems to be at least modest. In the PRIMA trial, the most frequent toxicities reported were grades 2-4 infection, occurring in 39\% patients assigned to the MR arm, and in $24 \%$ on the observation arm (risk ratio 1.62; 95\% CI: $1.35-1.96$; $P<0.0001$ ). However, the rate of grades 3-4 infection was similar between both groups, $4 \%$ for MR versus 1\% for observation. Most of the infections reported in both groups were bronchitis, upper respiratory tract infections, sinusitis, and urinary tract infections. ${ }^{56,57}$ The MAXIMA study, a Phase IIIb of MR in the daily care setting, reported a similar infection rate of $4.1 \%$ among the 545 patients included. ${ }^{63}$ On the other hand, the long-term outcome report of the EORTC 20981 study did find that patients receiving MR experienced significantly more grades 3-4 infections than those in the observational group $(9.7 \%$ versus $2.4 \%, P=0.01)$. This difference was in part attributed to the increased rate of grades 3-4 neutropenia in the MR arm, $11.5 \%$ versus $6 \%$ in the observation arm. ${ }^{61}$ An updated meta-analysis of nine trials (2,586 FL patients) comparing MR with no maintenance showed a significant increase in infections of all grades (pooled risk ratio $=1.67$; 95\% CI: 1.40-2.00). The risk was even larger when only grades 3-4 infection were analyzed (pooled risk ratio $=3.55$; 95\% CI: $1.88-6.69) .{ }^{64}$

Impairment on the immune system has been suggested as a predisposing factor for infection in patients receiving rituximab therapy. Prolonged neutropenia and late-onset neutropenia have been described as secondary effects of rituximab therapy, ${ }^{65,66}$ although long-term data about the impact of MR in such complications have not been studied so far. In the SAKK 35/98 study, patients assigned to MR took 6 months longer to recover the value of circulating B-cells to baseline values than patients assigned to observation. Serum IgG and IgA levels remained unchanged in both groups during therapy; however IgM levels decreased to $73 \%$ after 1 year in the MR group, while in the observational arm the median level got back to $100 \%(P=0.007) .{ }^{67}$ In the PRIMA trial a slight decrease in serum concentrations of immunoglobulins isotypes was observed from baseline to the end of maintenance phase in the MR group, although they did not differ significantly between both groups. ${ }^{57}$ Similar results were observed in the EORTC 20981 trial where patients randomized to MR did not have lower levels of serum immunoglobulins isotypes compared with those in the observation group. ${ }^{60}$ In a retrospective analysis, investigators from the Memorial Sloan Kettering Cancer Center reported that the rate of B-cell lymphoma patients developing hypogammaglobulinemia after rituximab was $38.5 \%$, and
$6.6 \%$ of patients required IV immune globulin administration for symptomatic hypogammaglobulinemia. The risk was greater in patients receiving MR. ${ }^{68}$

The occurrence of secondary malignancies (SM) in patients receiving immunosuppressive treatments is another point of concern to consider with the use of MR in FL. The data regarding SM from main studies of MR are not fully described; however, it seems to be similar to that described in other studies of CT treated patients. In the EORTC 20981 trial, the reported rate of SM was $8 \%$ in the observational group and 5\% in the MR group. ${ }^{61}$ The 10 -year follow-up of the SAKK 35/98 study reports an incidence of 23 second tumors in 151 patients (15\%), equally distributed among the two study arms. ${ }^{15}$ Updated 6-year follow-up of the PRIMA trial presented at the 2013 meeting of the American Society of Hematology reported that SM was the main cause of death in 19 and 5 patients assigned to the observation and MR arms, respectively. ${ }^{57}$

Delayed pulmonary damage, mainly in the form of interstitial lung disease has been described as another potential adverse effect of rituximab. ${ }^{69}$ The real incidence of this form of pulmonary toxicity is unknown. In 2003, in reply to a letter describing two NHL patients developing interstitial pneumonitis after rituximab therapy, the manufacturers reported that the calculated incidence was less than $0.03 \%$, with more than 300,000 patients worldwide exposed to rituximab. ${ }^{70}$ Probably, this incidence may be higher due to the number of unreported cases and those misdiagnoses as lung infections.

An important issue for clinicians is the risk of fulminant hepatitis resulting from hepatitis B virus (HBV) reactivation following rituximab therapy. In 2013, the US Food and Drug Administration added new "black box" warning information for rituximab (and for ofatumumab, another anti-CD20 mAb) about the risk of reactivation of $\mathrm{HBV}$ infection. ${ }^{71}$ Screening for HBV with hepatitis B surface antigen (HbsAg) and antihepatitis B core antibody ( $\mathrm{HbcAb})$, should be performed in all patients prior to the administration of rituximab. ${ }^{72}$ High-risk patients for $\mathrm{HBV}$ reactivation are chronic carriers (HbsAgpositive) as well as those with an occult infection or history of resolved hepatitis $\mathrm{B}$ (HbcAb-positive). ${ }^{72,73}$ More than $50 \%$ of patients with $\mathrm{HbsAg-positive} \mathrm{and} \mathrm{nearly} \mathrm{30 \% -40 \%}$ with $\mathrm{HbcAb}$-positive may present $\mathrm{HBV}$ reactivation during rituximab containing therapy, leading to $4 \%-5 \%$ death if no previous antiviral prophylaxis is administered. ${ }^{74,75}$ For those patients at risk, the recommendation is to start prophylaxis 7 days before the first rituximab dose and to continue beyond 1 year after the last dose, preferably with entecavir 
or tenofovir. ${ }^{72,76,77}$ On the other hand, the real incidence of hepatitis $\mathrm{C}$ virus reactivation after rituximab therapy is not clear, since this association has rarely been reported. Regarding patients treated with MR, data about hepatitis C virus reactivation are lack. ${ }^{78,79}$

Progressive multifocal leukoencephalopathy (PML) is an infrequent but almost uniformly lethal complication related to the use of rituximab and other immunosuppressive therapies. PML is a progressive demyelinating disorder of the central nervous system, due to the reactivation of a latent infection of the John Cunningham polyomavirus. ${ }^{80,81}$ The mortality rate is as high as $84 \%-100 \%$. In the largest series published to date involving $57 \mathrm{HIV}$-negative patients treated with rituximab, the mortality rate was $90 \% .{ }^{82}$ In this study, the median time for the development of PML after the last dose of rituximab was 5.5 months, and the median time to death after PML diagnosis was 2 months. Approximately $92 \%$ of the adult population is John Cunningham polyomavirus-seropositive, although PML tends to occur in patients with cellular immunosuppression, mainly in those affected by the HIV. ${ }^{81}$ In 2006, the labeling for rituximab was updated to include "black box" warning information about the risk of PML ${ }^{83}$ In a review of 2012, rituximab therapy had been associated with 157 cases of PML with an estimated two million doses administered, leading to an estimated event rate of 1:30,000. ${ }^{81} \mathrm{~A}$ high degree of awareness for PML is required when evaluating a rituximab treated patient who presents with new neurologic symptoms, aiming to set a prompt diagnosis in an attempt to avoid irreversible neurologic damage.

In summary, the long-term efficacy of MR confirms initial reports improving the outcomes of patients with FL. No new or unexpected safety findings have been described and the adverse events mentioned above are infrequent. Currently, there are two ongoing studies evaluating the long-term safety and efficacy of rituximab with prolonged administrations. The SAKK 35/03 trial $^{84}$ is comparing long-term MR for 5 years with a short-term maintenance of 8 months in patients with untreated or R/R FL after induction with four weekly rituximab, whereas the MAINTAIN trial ${ }^{85}$ compares 2 versus 4 years of MR in patients with $\mathrm{FL}$ after an induction regimen with $\mathrm{B}-\mathrm{R}$. On the other hand, longer follow-up of the RESORT trial will also provide us information about the long-term safety of MR.

\section{Effect of MR on QoL}

The effect of MR on the QoL has been assessed in various studies. ${ }^{39,40,56,86-88}$ Witzens-Harig et al ${ }^{86}$ first analyzed prospectively the impact of MR on QoL in 91 patients with NHL, among which 16 had FL. After completing induction therapy, patients were randomized to either MR every 3 months for 2 years or observation. The QoL of the patient in both study arms was analyzed using specific questionnaires (EORTC QLQ-30, EQ-5D, and EQ-5D VAS) that overall quantified health-related aspect. As a result, no statistically significant differences between MR and observation were found. Therefore, the authors concluded that MR therapy seems to be safe and does not affect QoL in this patient population. ${ }^{86}$ Walker et al ${ }^{87}$ performed a retrospective review at seven community oncology practices in the USA with the primary objective of examining symptom burden and QoL in patients with FL undergoing MR compared with observation. Health-related QoL was measured using Patient Care Monitor assessment, an electronic instrument validated for the symptomatic and functional evaluation of patients with cancer. Symptoms reported by patients were similar in both groups without a negative impact in QoL due to maintenance treatment with rituximab. In fact, psychological symptoms improved when the patients received active treatment with rituximab during the maintenance period. ${ }^{87}$ The two major randomized studies directly comparing WW versus active treatment with rituximab (with or without MR) in patients with LTB-FL, had tried to clarify not only the efficacy of treatment with rituximab but also the impact on QoL. ${ }^{39,40}$ In the Intergroup study, there was no negative effect on QoL between the patients treated in the rituximab arm (induction and maintenance) and the patients in the WW arm. Moreover, patients in the MR group, experienced a significant improvement in the Mental Adjustment to Cancer scores feeling these more in control of their disease and less anxious than those in the WW arm. ${ }^{39}$ In the RESORT trial, patients were randomized to MR every 3 months or rituximab retreatment at progression. ${ }^{40}$ Anxiety and QoL data were analyzed throughout the study and have recently been published separately by Wagner et al. ${ }^{88}$ Illness-related anxiety, general anxiety, and health-related QoL were similar in both groups. The patients assigned to retreatment at progression did not experience higher anxiety during the surveillance period than those receiving active treatment with rituximab. Accordingly, the authors conclude that relapse is not associated with emotional distress if the recurrence will be immediately re treated with a well-admitted therapy avoiding, moreover overtreatment. They also noted that regardless of the treatment strategy, coping style (active versus avoidant) interferes with the emotional well-being, emphasizing the importance of psychological intervention in patients who need it. ${ }^{88}$ Finally, the effectiveness of MR in HTB-FL patients responding to R-CT was evaluated in 
the PRIMA study. Assessment of QoL among participants was one of the secondary endpoints of the study. Patients assigned to observation after first-line treatment and those who received MR for 2 years completed a series of QoLrelated questionnaires during the maintenance period. In line with the studies mentioned above, there were no differences in the EORTC QLQ-C30 global health status mean scores or in the mean adjusted FACT-G total scores at the end of treatment between both groups. ${ }^{56}$

Consequently, it seems that MR does not impair QoL in patients with advanced FL. Therefore, in our opinion, the decision to adopt the MR strategy should not be subjected to this fact.

New formulation of SC rituximab may improve QoL in patients with CD20+ NHL as highlighted by preferences and satisfaction questionnaires in the PrefMab and MabCute studies when compared with the IV administration. ${ }^{89,90}$

\section{Conclusion, place in therapy}

Two decades after the first Phase I clinical trials, rituximab remains the cornerstone in the therapy of B-cell lymphomas. The prognosis of FL has been significantly improved with the incorporation of rituximab to CT regimens in both the first-line and the relapsed setting. The old paradigm that considered FL as an incurable condition is changing, and rituximab is being used increasingly in both the localized disease and in the first-line treatment of advanced LTB-FL where traditional management has been the WW strategy. Induction $\mathrm{R}-\mathrm{CT}$ regimens such as R-CHOP, B-R, or R-CVP are among the most used in the first-line treatment of HTB-FL. The adoption of maintenance treatment with rituximab after the first-line substantially improves results in terms of response rate, EFS and PFS with no relevant increase in toxicity. Longterm results of the PRIMA trial confirm MR every 2 months for 2 years as a standard approach after induction R-CT in advanced HTB-FL. Clinicians involved in the management of B-cell lymphomas have learned to recognize and handle the less common and late side effects that have emerged with the widespread use of rituximab, such as reactivation of HBV or the life-threatening PML among others.

The optimal duration of MR will be clarified in the coming years with the results of ongoing studies, and whether maintenance for 4 or 5 years will be able to further improve the results will be determined. In the meantime, improved results are being obtained in clinical trials with a number of newgeneration mAbs targeting the CD-20 antigen such as obinutuzumab (GA101, a new type II, humanized anti-CD20 mAb), ofatumumab, or veltuzumab. The new formulation of SC ritux- imab represents the next step on the way to improve comfort in the administration and QoL of patients with FL.

\section{Disclosure}

The authors report no conflicts of interest in this work.

\section{References}

1. Plosker GL, Figgitt DP. Rituximab: a review of its use in non-Hodgkin's lymphoma and chronic lymphocytic leukaemia. Drugs. 2003;63(8): 803-843.

2. Reff ME, Carner K, Chambers KS, et al. Depletion of B cells in vivo by a chimeric mouse human monoclonal antibody to CD20. Blood. 1994; 83(2):435-445.

3. Coiffier B. Rituximab therapy in malignant lymphoma. Oncogene. 2007;26(25):3603-3613.

4. Abdulla NE, Ninan MJ, Markowitz AB. Rituximab: current status as therapy for malignant and benign hematologic disorders. BioDrugs. 2012;26(2):71-82.

5. Anderson JR, Armitage JO, Weisenburger DD. Epidemiology of the non-Hodgkin's lymphomas: distributions of the major subtypes differ by geographic locations. Non-Hodgkin's Lymphoma Classification Project. Ann Oncol. 1998;9(7):717-720.

6. Campo E, Swerdlow SH, Harris NL, et al. The 2008 WHO classification of lymphoid neoplasms and beyond: evolving concepts and practical applications. Blood. 2011;117(19):5019-5032.

7. Tan D, Horning SJ, Hoppe RT, et al. Improvements in observed and relative survival in follicular grade 1-2 lymphoma during 4 decades: the Stanford University experience. Blood. 2013;122(6):981-987.

8. Golay JT, Clark EA, Beverley PC. The CD20 (Bp35) antigen is involved in activation of B cells from the G0 to the G1 phase of the cell cycle. J Immunol. 1985;135(6):3795-3801.

9. LiH, Ayert LM, Lytton J, Deans JP. Store-operated cation entry mediated by CD20 in membrane rafts. J Biol Chem. 2003;278(43):42427-42434.

10. Manches $\mathrm{O}$, Lui G, Chaperot L, et al. In vitro mechanisms of action of rituximab on primary non-Hodgkin lymphomas. Blood. 2003;101(3): 949-954.

11. Maloney DG, Smith B, Rose A. Rituximab: mechanism of action and resistance. Semin Oncol. 2002;29(Suppl 2):2-9.

12. Cartron G, Dacheux L, Salles G, et al. Therapeutic activity of humanized anti-CD20 monoclonal antibody and polymorphism in $\mathrm{IgG} \mathrm{Fc}$ receptor FcgammaRIIIa gene. Blood. 2002;99(3):754-758.

13. Koene HR, Kleijer M, Algra J, et al. Fc gammaRIIIa-158V/F polymorphism influences the binding of IgG by natural killer cell $\mathrm{Fc}$ gammaRIIIa, independently of the $\mathrm{Fc}$ gammaRIIIa-48L/R/H phenotype. Blood. 1997;90(3):1109-1114.

14. Weng WK, Levy R. Two immunoglobulin G fragment C receptor polymorphisms independently predict response to rituximab in patients with follicular lymphoma. J Clin Oncol. 2003;21(21):3940-3947.

15. Martinelli G, Schmitz SF, Utiger U, et al. Long-term follow-up of patients with follicular lymphoma receiving single-agent rituximab at two differen schedules in trial SAKK 35/98. J Clin Oncol. 2010;28(29):4480-4484.

16. Venugopal P, Sivaraman S, Huang XK, et al. Effects of cytokines on CD20 antigen expression on tumor cells from patients with chronic lymphocytic leukemia. Leuk Res. 2000;24(5):411-415.

17. van der Kolk LE, Grillo-Lopez AJ, Baars JW, et al. Complement activation plays a key role in the side-effects of rituximab treatment. Br J Haematol. 2001;115(4):807-811.

18. Kennedy AD, Beum PV, Solga MD, et al. Rituximab infusion promotes rapid complement depletion and acute CD20 loss in chronic lymphocytic leukemia. J Immunol. 2004;172(5):3280-3288.

19. Abulayha A, Bredan A, El Enshasy H, Daniels I. Rituximab: modes of action, remaining dispute and future perspective. Future Oncol. 2014;10(15):2481-2492.

20. Rodriguez J, Gutierrez A. Pharmacokinetic properties of rituximab. Rev Recent Clin Trials. 2008;3(1):22-30. 
21. Jazirehi AR, Gan XH, De Vos S, et al. Rituximab (anti-CD20) selectively modifies Bcl-xL and apoptosis protease activating factor-1 (Apaf-1) expression and sensitizes human non-Hodgkin's lymphoma B cell lines to paclitaxel-induced apoptosis. Mol Cancer Ther. 2003;2(11): 1183-1193.

22. Keating GM. Rituximab: a review of its use in chronic lymphocytic leukaemia, low-grade or follicular lymphoma and diffuse large B-cell lymphoma. Drugs. 2010;70(11):1445-1476.

23. Mangel J, Buckstein R, Imrie K, et al. Pharmacokinetic study of patients with follicular or mantle cell lymphoma treated with rituximab as "in vivo purge" and consolidative immunotherapy following autologous stem cell transplantation. Ann Oncol. 2003;14(5):758-765.

24. Maloney DG, Liles TM, Czerwinski DK, et al. Phase I clinical trial using escalating single-dose infusion of chimeric anti-CD20 monoclonal antibody (IDEC-C2B8) in patients with recurrent B-cell lymphoma. Blood. 1994;84(8):2457-2466.

25. Maloney DG, Grillo-Lopez AJ, Bodkin DJ, et al. IDEC-C2B8: results of a phase I multiple-dose trial in patients with relapsed non-Hodgkin's lymphoma. J Clin Oncol. 1997;15(10):3266-3274.

26. McLaughlin P, Grillo-Lopez AJ, Link BK, et al. Rituximab chimeric anti-CD20 monoclonal antibody therapy for relapsed indolent lymphoma: half of patients respond to a four-dose treatment program. J Clin Oncol. 1998;16(8):2825-2833.

27. Berinstein NL, Grillo-Lopez AJ, White CA, et al. Association of serum rituximab (IDEC-C2B8) concentration and anti-tumor response in the treatment of recurrent low-grade or follicular non-Hodgkin's lymphoma. Ann Oncol. 1998;9(9):995-1001.

28. Piro LD, White CA, Grillo-Lopez AJ, et al. Extended Rituximab (anti-CD20 monoclonal antibody) therapy for relapsed or refractory low-grade or follicular non-Hodgkin's lymphoma. Ann Oncol. 1999;10(6):655-661.

29. De Cock E, Kritikou P, Tao S, et al. Time savings with rituximab subcutaneous (SC) injection vs rituximab intravenous (IV) infusion: final analysis from a time-and-motion study in 8 countries. Blood. 2013;122(21): abstr 1742 .

30. Salar A, Bouabdallah R, McIntyre C, et al. A two-stage phase Ib study to investigate the pharmacokinetics, safety and tolerability of subcutaneous rituximab in patients with follicular lymphoma as part of maintenance treatment. Blood. 2010;116(21): abstr 2858.

31. Frost GI. Recombinant human hyaluronidase (rHuPH20): an enabling platform for subcutaneous drug and fluid administration. Expert Opin Drug Deliv. 2007;4(4):427-440.

32. Salar A, Avivi I, Bittner B, et al. Comparison of subcutaneous versus intravenous administration of rituximab as maintenance treatment for follicular lymphoma: results from a two-stage, phase IB study. J Clin Oncol. 2014;32(17):1782-1791.

33. Davies A, Merli F, Mihaljevic B, et al. Pharmacokinetics and safety of subcutaneous rituximab in follicular lymphoma (SABRINA): stage 1 analysis of a randomised phase 3 study. Lancet Oncol. 2014;15(3): 343-352.

34. Brice $\mathrm{P}$, Bastion Y, Lepage E, et al. Comparison in low-tumor-burden follicular lymphomas between an initial no-treatment policy, prednimustine, or interferon alfa: a randomized study from the Groupe d'Etude des Lymphomes Folliculaires. J Clin Oncol. 1997;15(3): 1110-1117.

35. Ardeshna KM, Smith P, Norton A, et al. Long-term effect of a watch and wait policy versus immediate systemic treatment for asymptomatic advanced-stage non-Hodgkin lymphoma: a randomised controlled trial. Lancet. 2003;362(9383):516-522.

36. Young RC, Longo DL, Glatstein E, et al. The treatment of indolent lymphomas: watchful waiting $\mathrm{v}$ aggressive combined modality treatment. Semin Hematol. 1988;25(Suppl 2):11-16.

37. O'Brien ME, Easterbrook P, Powell J, et al. The natural history of low grade non-Hodgkin's lymphoma and the impact of a no initial treatment policy on survival. Q J Med. 1991;80(292):651-660.

38. Czuczman MS. Controversies in follicular lymphoma: "who, what, when, where, and why?" (not necessarily in that order!). Hematology Am Soc Hematol Educ Program. 2006:303-310.
39. Ardeshna KM, Qian W, Smith P, et al. Rituximab versus a watch-andwait approach in patients with advanced-stage, asymptomatic, non-bulky follicular lymphoma: an open-label randomised phase 3 trial. Lancet Oncol. 2014;15(4):424-435.

40. Kahl BS, Hong F, Williams ME, et al. Rituximab extended schedule or re-treatment trial for low-tumor burden follicular lymphoma: Eastern Cooperative Oncology Group protocol E4402. J Clin Oncol. 2014;32(28):3096-3102.

41. Hiddemann W, Kneba M, Dreyling M, et al. Frontline therapy with rituximab added to the combination of cyclophosphamide, doxorubicin, vincristine, and prednisone (CHOP) significantly improves the outcome for patients with advanced-stage follicular lymphoma compared with therapy with $\mathrm{CHOP}$ alone: results of a prospective randomized study of the German Low-Grade Lymphoma Study Group. Blood. 2005;106(12):3725-3732.

42. Herold M, Haas A, Srock S, et al. Rituximab added to first-line mitoxantrone, chlorambucil, and prednisolone chemotherapy followed by interferon maintenance prolongs survival in patients with advanced follicular lymphoma: an East German Study Group Hematology and Oncology Study. J Clin Oncol. 2007;25(15):1986-1992.

43. Marcus R, Imrie K, Solal-Celigny P, et al. Phase III study of R-CVP compared with cyclophosphamide, vincristine, and prednisone alone in patients with previously untreated advanced follicular lymphoma. J Clin Oncol. 2008;26(28):4579-4586.

44. Salles G, Mounier N, De Guibert S, et al. Rituximab combined with chemotherapy and interferon in follicular lymphoma patients: results of the GELA-GOELAMS FL2000 study. Blood. 2008;112(13):4824-4831.

45. Federico M, Luminari S, Dondi A, et al. R-CVP versus R-CHOP versus R-FM for the initial treatment of patients with advanced-stage follicular lymphoma: results of the FOLL05 trial conducted by the Fondazione Italiana Linfomi. J Clin Oncol. 2013;31(12):1506-1513.

46. Rummel MJ, Niederle N, Maschmeyer G, et al. Bendamustine plus rituximab versus $\mathrm{CHOP}$ plus rituximab as first-line treatment for patients with indolent and mantle-cell lymphomas: an open-label, multicentre, randomised, Phase 3 non-inferiority trial. Lancet. 2013;381(9873): 1203-1210.

47. Morschhauser F, Radford J, Van Hoof A, et al. 90Yttrium-ibritumomab tiuxetan consolidation of first remission in advanced-stage follicular non-Hodgkin lymphoma: updated results after a median follow-up of 7.3 years from the International, Randomized, Phase III First-Line Indolent trial. J Clin Oncol. 2013;31(16):1977-1983.

48. Press OW, Unger JM, Rimsza LM, et al. Phase III randomized Intergroup trial of $\mathrm{CHOP}$ plus rituximab compared with $\mathrm{CHOP}$ chemotherapy plus (131) iodine-tositumomab for previously untreated follicular non-Hodgkin lymphoma: SWOG S0016. J Clin Oncol. 2013; 31(3):314-320

49. Al Khabori M, de Almeida JR, Guyatt GH, Kuruvilla J, Crump M. Autologous stem cell transplantation in follicular lymphoma: a systematic review and meta-analysis. $J$ Natl Cancer Inst. 2012;104(1):18-28.

50. Wang B, Ren C, Zhang W, et al. Intensified therapy followed by autologous stem-cell transplantation (ASCT) versus conventional therapy as first-line treatment of follicular lymphoma: a meta-analysis. Hematol Oncol. 2013;31(1):29-33.

51. Montoto S, Corradini P, Dreyling M, et al. Indications for hematopoietic stem cell transplantation in patients with follicular lymphoma: a consensus project of the EBMT-Lymphoma Working Party. Haematologica. 2013;98(7):1014-1021.

52. Solal-Celigny P, Lepage E, Brousse N, et al. Recombinant interferon alfa- $2 \mathrm{~b}$ combined with a regimen containing doxorubicin in patients with advanced follicular lymphoma. Groupe d'Etude des Lymphomes de l'Adulte. N Engl J Med. 1993;329(22):1608-1614.

53. Rohatiner AZ, Gregory WM, Peterson B, et al. Meta-analysis to evaluate the role of interferon in follicular lymphoma. J Clin Oncol. 2005;23(10):2215-2223.

54. Ghielmini M, Schmitz SF, Cogliatti SB, et al. Prolonged treatment with rituximab in patients with follicular lymphoma significantly increases event-free survival and response duration compared with the standard weekly $\times 4$ schedule. Blood. 2004;103(12):4416-4423. 
55. Hochster H, Weller E, Gascoyne RD, et al. Maintenance rituximab after cyclophosphamide, vincristine, and prednisone prolongs progression-free survival in advanced indolent lymphoma: results of the randomized Phase III ECOG1496 study. J Clin Oncol. 2009;27(10):1607-1614.

56. Salles G, Seymour JF, Offner F, et al. Rituximab maintenance for 2 years in patients with high tumour burden follicular lymphoma responding to rituximab plus chemotherapy (PRIMA): a Phase 3, randomised controlled trial. Lancet. 2011;377(9759):42-51.

57. Salles GA, Seymour JF, Feugier P, et al. Updated 6 year follow-up of the PRIMA study confirms the benefit of 2-year rituximab maintenance in follicular lymphoma patients responding to frontline immunochemotherapy. Blood. 2013;122(21): abstr 509

58. Vitolo U, Ladetto M, Boccomini C, et al. Rituximab maintenance compared with observation after brief first-line R-FND chemoimmunotherapy with rituximab consolidation in patients age older than 60 years with advanced follicular lymphoma: a Phase III randomized study by the Fondazione Italiana Linfomi. J Clin Oncol. 2013;31(27):3351-3359.

59. Forstpointner R, Dreyling M, Repp R, et al. The addition of rituximab to a combination of fludarabine, cyclophosphamide, mitoxantrone (FCM) significantly increases the response rate and prolongs survival as compared with FCM alone in patients with relapsed and refractory follicular and mantle cell lymphomas: results of a prospective randomized study of the German Low-Grade Lymphoma Study Group. Blood. 2004;104(10):3064-3071.

60. van Oers MH, Klasa R, Marcus RE, et al. Rituximab maintenance improves clinical outcome of relapsed/resistant follicular non-Hodgkin lymphoma in patients both with and without rituximab during induction results of a prospective randomized phase 3 intergroup trial. Blood 2006;108(10):3295-3301

61. van Oers MH, Van Glabbeke M, Giurgea L, et al. Rituximab maintenance treatment of relapsed/resistant follicular non-Hodgkin's lymphoma: long-term outcome of the EORTC 20981 phase III randomized intergroup study. J Clin Oncol. 2010;28(17):2853-2858.

62. Forstpointner R, Unterhalt M, Dreyling M, et al. Maintenance therapy with rituximab leads to a significant prolongation of response duration after salvage therapy with a combination of rituximab, fludarabine, cyclophosphamide, and mitoxantrone (R-FCM) in patients with recurring and refractory follicular and mantle cell lymphomas: results of a prospective randomized study of the German Low Grade Lymphoma Study Group (GLSG). Blood. 2006;108(13):4003-4008.

63. Witzens-Harig M, Foá R, Di Rocco A, et al. Maintenance with rituximab is safe and not associated with severe or uncommon infections in patients with follicular lymphoma: results from the phase IIIb MAXIMA study. Ann Hematol. 2014;93(10):1717-1724.

64. Vidal L, Gafter-Gvili A, Salles G, et al. Rituximab maintenance for the treatment of patients with follicular lymphoma: an updated systematic review and meta-analysis of randomized trials. J Natl Cancer Inst. 2011;103(23):1799-1806.

65. Voog E, Morschhauser F, Solal-Céligny P. Neutropenia in patients treated with rituximab. $N$ Engl J Med. 2003;348(26):2691-2694.

66. Tesfa D, Palmblad J. Late-onset neutropenia following rituximab therapy: incidence, clinical features and possible mechanisms. Expert Rev Hematol. 2011;4(6):619-625.

67. Ghielmini M, Rufibach K, Salles G, et al. Single agent rituximab in patients with follicular or mantle cell lymphoma: clinical and biological factors that are predictive of response and event-free survival as well as the effect of rituximab on the immune system: a study of the Swiss Group for Clinical Cancer Research (SAKK). Ann Oncol. 2005;16(10): 1675-1682.

68. Casulo C, Maragulia J, Zelenetz AD. Incidence of hypogammaglobulinemia in patients receiving rituximab and the use of intravenous immunoglobulin for recurrent infections. Clin Lymphoma Myeloma Leuk. 2013;13(2):106-111.

69. Liu X, Hong XN, Gu YJ, et al. Interstitial pneumonitis during rituximabcontaining chemotherapy for non-Hodgkin lymphoma. Leuk Lymphoma. 2008;49(9):1778-1783.
70. Burton C, Kaczmarski R, Jan-Mohamed R. Interstitial pneumonitis related to rituximab therapy. N Engl J Med. 2003;348(26):2690-2691.

71. US Food and Drug Administration. FDA Drug Safety Communication: Boxed Warning and New Recommendations to Decrease Risk of Hepatitis B Reactivation with the Immune-suppressing and Anti-cancer Drugs Arzerra (Ofatumumab) and Rituxan (Rituximab). Silver Spring, MD: US Food and Drug Administration; 2013. Available at: http://www.fda.gov/ Drugs/DrugSafety/ucm366406.htm. Accessed July 1, 2015.

72. Hwang JP, Somerfield MR, Alston-Johnson DE, et al. Hepatitis B virus screening for patients with cancer before therapy: American Society of Clinical Oncology provisional clinical opinion update. J Clin Oncol. 2015;33(19):2212-2220.

73. Seto WK, Chan TS, Hwang YY, et al. Hepatitis B reactivation in patients with previous hepatitis B virus exposure undergoing rituximabcontaining chemotherapy for lymphoma: a prospective study. J Clin Oncol. 2014;32(33):3736-3743.

74. Yeo W, Chan TC, Leung NW, et al. Hepatitis B virus reactivation in lymphoma patients with prior resolved hepatitis B undergoing anticancer therapy with or without rituximab. J Clin Oncol. 2009;27(4): 605-611.

75. Zurawska U, Hicks LK, Woo G, et al. Hepatitis B virus screening before chemotherapy for lymphoma: a cost-effectiveness analysis. $J$ Clin Oncol. 2012;30(26):3167-3173.

76. Huang YH, Hsiao LT, Hong YC, et al. Randomized controlled trial of entecavir prophylaxis for rituximab-associated hepatitis B virus reactivation in patients with lymphoma and resolved hepatitis B. J Clin Oncol. 2013;31(22):2765-2772.

77. Viganò M, Mangia G, Lampertico P. Management of patients with overt or resolved hepatitis B virus infection undergoing rituximab therapy. Expert Opin Biol Ther. 2014;14(7):1019-1031.

78. Nosotti L, D’Andrea M, Pitidis A, et al. Hepatitis C virus infection prevalence and liver dysfunction in a cohort of B-cell non-Hodgkin's lymphoma patients treated with immunochemotherapy. Scand J Infect Dis. 2012;44(1):70-73.

79. Yazici O, Sendur MA, Aksoy S. Hepatitis C virus reactivation in cancer patients in the era of targeted therapies. World J Gastroenterol. 2014;20(22):6716-6724.

80. Brooks BR, Walker DL. Progressive multifocal leukoencephalopathy. Neurol Clin. 1984;2(2):299-313.

81. Zaheer F, Berger JR. Treatment-related progressive multifocal leukoencephalopathy: current understanding and future steps. Ther $A d v$ Drug Safety. 2012;3(5):227-239.

82. Carson KR, Evens AM, Richey EA, et al. Progressive multifocal leukoencephalopathy after rituximab therapy in HIV-negative patients: a report of 57 cases from the Research on Adverse Drug Events and Reports project. Blood. 2009;113(20):4834-4840.

83. US Food and Drug Administration. Information for Healthcare Professionals: Rituximab (marketed as Rituxan). Silver Spring, MD: US Food and Drug Administration; 2006. Available from: http://www. fda.gov/Drugs/DrugSafety/PostmarketDrugSafetyInformationforPatientsandProviders/ucm126519.htm. Accessed July 1, 2015.

84. Taverna CJ, Martinelli G, Hitz F, et al. Rituximab maintenance treatment for a maximum of 5 years in follicular lymphoma: results of the randomized Phase III trial SAKK 35/03. Blood. 2013;122(21): abstr 508.

85. Rummel MJ, Viardot A, Greil R, et al. Bendamustine plus rituximab followed by rituximab maintenance for patients with untreated advanced follicular lymphomas. Results from the StiL NHL 7-2008 trial (MAINTAIN trial) (ClinicalTrials.gov Identifier: NCT00877214). Blood. 2014;124(21): abstr 3052.

86. Witzens-Harig M, Reiz M, Heiss C, et al. Quality of life during maintenance therapy with the anti-CD20 antibody rituximab in patients with B cell non-Hodgkin's lymphoma: results of a prospective randomized controlled trial. Ann Hematol. 2009;88(1):51-57.

87. Walker MS, Stepanski EJ, Reyes C, et al. Symptom burden and quality of life in patients with follicular lymphoma undergoing maintenance treatment with rituximab compared with observation. Ther Adv Hematol. 2011;2(3):129-139. 
88. Wagner LI, Zhao F, Hong F, et al. Anxiety and health-related quality of life among patients with low-tumor burden non-Hodgkin lymphoma randomly assigned to two different rituximab dosing regimens: results from ECOG trial E4402 (RESORT). J Clin Oncol. 2015;33(7):740-748.

89. Rule S, Briones J, Smith R, et al. Preference for rituximab subcutaneous (SC) and intravenous (IV) among patients with CD20+ non-Hodgkin's lymphoma (NHL) completing the Rasq Measure in randomized phase III studies PrefMab and MabCute. Value Health. 2014;17(7):A537.
90. Rummel M, Kim TM, Plenteda C, et al. Patient preference for subcutaneous or intravenous administration of rituximab in previously untreated CD20+ non-Hodgkin lymphoma: interim data from the PrefMab study. Haematologica. 2014;99(Suppl 1):152-153.

\section{Publish your work in this journal}

Cancer Management and Research is an international, peer-reviewed open access journal focusing on cancer research and the optimal use of preventative and integrated treatment interventions to achieve improved outcomes, enhanced survival and quality of life for the cancer patient. The journal welcomes original research, clinical \& epidemiological studies, reviews \& evaluations, guidelines, expert opinion \& commentary, case reports \& extended reports. The manuscript management system is completely online and includes a very quick and fair peerreview system, which is all easy to use. Visit http://www.dovepress.com/ testimonials.php to read real quotes from published authors. 